\title{
PREVALENCIA DE ENFERMEDAD PERIODONTAL EN ESCOLARES DE 12 AÑOS DE CHIQUINTAD CUENCA (Azuay, Ecuador).
}

\section{PREVALENCE OF PERIODONTAL DISEASE IN 12 YEARS OLD SCHOOLCHILDREN FROM CHIQUINTAD CUENCA (Azuay, Ecuador).}

\author{
Naranjo-Rodríguez Andrea Paola ${ }^{1}$ Vásquez-Palacios Ana Cristina ${ }^{2}$ Gómez-Aguilar Mishell Estephanía $^{3 *}$ \\ ${ }^{1}$ Odontóloga de consulta particular.Ecuador. \\ ${ }^{2}$ Catedrática de la Facultad de Odontología de la Universidad Católica de Cuenca. Ecuador. ${ }^{3}$ Odontóloga, Universidad de \\ Cuenca,Ecuador. \\ mishu-gomez@hotmail.com
}

\section{Resumen}

Objetivo: Determinar la prevalencia de enfermedad periodontal en escolares de 12 años pertenecientes a la Parroquia Chiquintad del cantón Cuenca, Ecuador, en el año 2016.Materiales y Métodos: 100 escolares de 12 años de instituciones públicas de la parroquia de Chiquintad en el cantón Cuenca (Azuay, Ecuador) fueron seleccionados y examinados clínicamente por investigadores calibrados para evaluar enfermedad periodontal, empleándose el índice periodontal de Russell, además de aplicar una encuesta. Resultados:Los hallazgos revelaron que la prevalencia de enfermedad periodontal fue del $71 \%$. El promedio del IPR para el grupo de estudio fue de 0,252 (0,371) correspondiente a tejido de soporte sano. Conclusión: Existe una alta prevalencia de enfermedad periodontal en los adolescentes de 12 años pertenecientes a las instituciones públicas rurales de Cuenca, Ecuador. Los datos obtenidos podrían permitir diseñar mejores estrategias de manejo de los recursos destinados al nivel primario de salud.

Palabras clave: Enfermedades periodontales, adolescente, prevalencia . (DeCS, BIREME).

\begin{abstract}
Aim: To determine the prevalence of periodontal disease in 12-year-old school children belonging to the Chiquintad Parish of Cuenca, Ecuador, in 2016. Materials and Methods: 100 schoolchildren from 12 years of public institutions of the parish of Chiquintad in the canton of Cuenca (Azuay, Ecuador) were selected and clinically examined by researchers calibrated to evaluate periodontal disease, using Russell's periodontal index, in addition to applying a survey. textbf Results: The findings revealed that the prevalence of periodontal disease was $71 \%$. The average of the IPR for the study group was 0.252 (0.371) corresponding to healthy support tissue. CONCLUSION: There is a high prevalence of periodontal disease in 12-year-old adolescents belonging to rural public institutions in Cuenca, Ecuador. The data obtained could allow to design better management strategies for the resources destined to the primary health level.
\end{abstract}

Key words: Periodontal Diseases, adolescent, prevalence (DeCS, BIREME).

\section{INTRODUCCIÓN}

Las enfermedades periodontales (EP) constituyen un grupo de condiciones inflamatorias crónicas que afectan a los tejidos de soporte y protección del diente, ${ }^{1}$ que se presentan frecuentemente en la población en general. ${ }^{2}$ Este tipo de enfermedades perjudican la calidad de vida de las personas al reducir la capacidad funcional de su aparato masticatorio, afectar su autoestima y limitar su capacidad de establecer relaciones sociales. ${ }^{3}$ Las periodontopatías afectan con mayor frecuencia a los habitantes de países en vías de desarrollo, en especial a aquellos de escasos recursos económicos. ${ }^{4,5}$

Según la Organización Mundial de la Salud (OMS), las enfermedades periodontales graves, que pueden desembocar en la pérdida de dientes, afectan aproximadamente a un $20 \%$ de los adultos de edad media (35-44 años), a nivel mundial. ${ }^{6}$ En cuanto a los niños y adolescentes, los datos epidemiológi- 
cos revelan una elevada prevalencia de la gingivitis en países desarrollados, llegando a superar, en algunos casos, el $80 \%$ del total de individuos estudiados. ${ }^{2}$ En relación con el grupo de adolescentes, estudios realizados en México ${ }^{7}$ Nicaragua $^{8} y$ Chile ${ }^{9}$ mostraron igualmente una alta prevalecía de enfermedades periodontales en escolares de 12 años, con valores que varían entre un $75 \%$ y $96 \%$, respectivamente.

Pese a su importancia como determinantes de problemas graves de salud, son escasos los trabajos que hasta la fecha se han publicado en torno al tema de las enfermedades periodontales en el Ecuador. Uno de ellos, basado en el estudio de escolares de 12 años de una zona urbana de la región de la Sierra, reveló una prevalencia de enfermedades periodontales del $74 \% .{ }^{10}$ Sin embargo, la información sigue siendo muy escasa y dispersa; por otra parte, no se han publicado resultados que se refieran a comunidades rurales. Por estas razones, el objetivo del presente estudio fue determinar la prevalencia de enfermedades periodontales en escolares de 12 años pertenecientes a la Parroquia Chiquintad del cantón Cuenca- Ecuador en el año 2016.

\section{MATERIALES Y MÉTODOS}

El estudio cumple con la normativa vigente propuesta por el grupo de expertos STROBE. ${ }^{11}$ Se trató de un estudio epidemiológico, descriptivo y de corte transversal. La población de estudio estuvo conformada por 121 estudiantes de 12 años de edad (cumplidos o por cumplir hasta el 31 de Diciembre del año 2016), matriculados en los tres centros educativos públicos de la Parroquia Chiquintad de la ciudad de Cuenca. Además de la edad, entre los criterios de inclusión se tomó en cuenta la firma de un consentimiento expreso, por parte de padres o representantes legales, para participar en este estudio. Fueron excluidos del estudio aquellos escolares que no aceptaron el examen bucal, que no presentaron el consentimiento informado firmado, que portaban aparatos ortodónticos, o presentaban enfermedades sistémicas, problemas de locomoción y/o alteraciones psicológicas.

El examen clínico fue realizado por un examinador, previamente calibrado por un especialista de la Universidad Católica de Cuenca (Kappa>80\%), empleando luz natural y espejo bucal estéril. Se utilizaron todas las técnicas y las barreras de protección para garantizar la seguridad tanto del paciente como del operador.

Para determinar el grado de la patología periodontal se empleó el índice periodontal de Russell (IPR), ${ }^{12}$ aplicándose exclusivamente los criterios clínicos, ${ }^{13}$ debido a su fácil reproducibilidad. De acuerdo con la severidad de la enfermedad, los valores de este índice para cada una de las piezas dentales examinadas fueron los siguientes: 0 (negativo, encía sin inflamación manifiesta); 1 (gingivitis leve, área de inflamación en encía libre, no circunscribe al diente); 2 ( gingivitis grave, inflamación gingival que rodea completamente al diente, sin ruptura evidente de la adherencia epitelial); 6 (gingivitis con bolsa periodontal, desprendimiento de la inserción epitelial, no hay interferencia en la función masticatoria normal, ni movilidad dentaria); y 8 (destrucción periodontal avanzada con pérdida de la función masticatoria, movilidad dentaria y migración patológica). Para el cálculo del promedio del índice de Russell de cada escolar se sumaron todos los valores correspondientes a cada uno de las piezas evaluadas y se dividieron por el número de dientes presentes, estableciendo el estado clínico periodontal de cada escolar como sigue: de 0 a 0,2: tejido de soporte sano; de 0,3 a 0,9: gingivitis simple; de 1,0 a 1,9: gingivitis severa o periodontitis inicial; de 2,0 a 4,9: enfermedad periodontal destructiva establecida; y de 5,0 a 8,0: enfermedad periodontal terminal. ${ }^{14}$ Para el presente estudio se consideró totalmente sanos a los pacientes que presentaron un valor promedio de IPR igual a cero. A la par se aplicó una encuesta a los mismos escolares para evaluar sus hábitos de higiene bucal.

Para el ingreso de los datos se utilizó el programa de libre acceso EPI INFO (v.7.2) y posteriormente se analizaron mediante el paquete estadístico R (v.3.1.1). El análisis estadístico proporcionó información de carácter descriptivo y analítico acerca de la condición periodontal, para caracterizar demográficamente a la población estudiada y para describir la condición periodontal de los participantes. Para determinar posibles diferencias entre los grupos estudiados de la tablas 1 y 2 , se aplicó el test de Chicuadrado con el cálculo del valor p basado en el modelo de Monte Carlo (con 10000 simulaciones). ${ }^{15}$ En la tabla 3 se aplicó el test de Chi-cuadrado de Pearson.

\section{RESULTADOS}

Del total de escolares de 12 años de edad susceptibles de ser incluidos en el estudio $(\mathrm{N}=121), 100$ adolescentes $(82,64 \%)$ cumplieron con los criterios de selección y fueron, por lo tanto, examinados, De estos, 47 (47\%) fueron de sexo masculino y $53(53 \%)$ de sexo femenino.

La prevalencia de enfermedad periodontal para todo el grupo fue de $71 \%$. En los escolares de sexo masculino fue de $70,2 \%$; mientras que en los de sexo femenino fue del $71,7 \%$, diferencia que no fue estadísticamente significativa (Chi-cuadrado=0,026; $\mathrm{p}=0,8702$ ).

En cuanto a la severidad de la enfermedad periodontal, al comparar los resultados de la evaluación clínica mediante el IPR mostró que no existen diferencias significativas en función del sexo de los escolares (Chi-cuadrado $=2,273$; $\mathrm{p}=0,935)$. Ninguno de los participantes presentó enfermedad periodontal terminal (Tabla 1). El promedio del IPR para todo el grupo de estudio fue de 0,252 ; discriminado de la siguiente manera: 0,238 para escolares de sexo femenino y 0,268 para escolares de sexo masculino.

Con respecto a los hábitos de higiene bucal, los resultados de la encuesta aplicada revelaron que un $86 \%$ de los escolares mencionan que realizan su cepillado dental entre 2 a 3 veces al día (Tabla 2). Así también, que apenas el 


\begin{tabular}{|c|c|c|c|c|c|c|}
\hline \multirow[b]{2}{*}{ Nivel de severidad } & \multicolumn{2}{|c|}{ FEMENINO } & \multicolumn{2}{|c|}{ MASCULINO } & \multicolumn{2}{|c|}{ TOTAL } \\
\hline & $\mathbf{n}$ & $\%$ & $\mathbf{n}$ & $\%$ & $\mathbf{n}$ & $\%$ \\
\hline Tejido de soporte sano $(0-0,2)$ & 41 & 77,4 & 35 & 74,5 & 76 & 76 \\
\hline Gingivitis simple $(0,3-0,9)$ & 7 & 13,2 & 5 & 10,6 & 12 & 12 \\
\hline Gingivitis severa o period. inicial $(1,0-1,9)$ & 4 & 7,5 & 7 & 14,9 & 11 & 11 \\
\hline $\begin{array}{l}\text { Enfermedad periodontal destructiva } \\
\text { establecida }(2,0-4,9)\end{array}$ & 1 & 1,9 & 0 & 0 & 1 & 1 \\
\hline Enfermedad periodontal terminal $(5,0-8,0)$ & 0 & 0 & 0 & 0 & 0 & 0 \\
\hline
\end{tabular}

Tabla 1. NIVEL DE SEVERIDAD DE ENFERMEDAD PERIODONTAL EN ESCOLARES DE 12 AÑOS DE LA PARROQUIA CHIQUINTAD (CUENCA, ECUADOR).

\begin{tabular}{ccccccc}
\hline $\begin{array}{c}\text { Cepillado diario } \\
\text { (Veces) }\end{array}$ & \multicolumn{2}{c}{ FEMENINO } & \multicolumn{2}{c}{ MASCULINO } & \multicolumn{2}{c}{ TOTAL } \\
\hline $\mathbf{n}$ & $\mathbf{\%}$ & $\mathbf{n}$ & $\mathbf{\%}$ & $\mathbf{n}$ & $\mathbf{\%}$ \\
\hline $\mathbf{2}$ & 4 & 7,5 & 5 & 10,6 & 9 & 9 \\
$\mathbf{3}$ & 17 & 32,1 & 20 & 42,6 & 37 & 37 \\
$\mathbf{4}$ & 30 & 56,6 & 19 & 40,4 & 49 & 49 \\
$\mathbf{5}$ & 2 & 3,8 & 2 & 4,3 & 4 & 4 \\
\hline
\end{tabular}

Tabla 2. NÚMERO DE VECES QUE LOS ESCOLARES INCLUIDOS EN EL ESTUDIO SE CEPILLAN AL DÍA.

$22 \%$ emplea seda dental. Llama la atención que el $2 \%$ de los escolares no tiene cepillo dental y el $3 \%$ de ellos lo comparte con algún miembro de su familia (Tabla 3).

\section{DISCUSIÓN}

Los resultados obtenidos durante la realización del presente estudio revelaron una prevalencia elevada de enfermedad periodontal $(71 \%)$ en adolescentes de 12 años pertenecientes a un sector rural en la Sierra Ecuatoriana. No obstante, la mayoría de estos escolares se encontraban en una etapa incipiente del desarrollo de la enfermedad.

Estudios similares, realizados en México ${ }^{7} \mathrm{Chile}^{9}$ en adolescentes del mismo grupo etario reportan valores muy parecidos al nuestro $(75,45 \%$ y $76,71 \%$, respectivamente). La similitud podría estar relacionada con el hecho de que, en todos los casos, se trata de adolescentes pertenecientes a comunidades rurales, con bajos niveles de ingresos y escaso acceso a los servicios de salud.
Por el contrario, en estudios realizados en adolescentes de 12 años de sectores rurales en Brasil ${ }^{16}$ y Cuba, ${ }^{17}$ los valores reportados $(30,5 \%$ y $16 \%$ respectivamente) son inferiores a los de nuestro estudio. Esto podría deberse a que Cuba presenta una cobertura nacional de atención en salud pública con énfasis en la prevención de enfermedades bucales, así como de promoción de la higiene bucal y conservación de la salud bucodental. ${ }^{18}$ Llama la atención la baja prevalencia de EP en el caso de Brasil: en efecto, se trata de un país que no tiene la misma cobertura de atención en salud bucodental por parte de instituciones públicas que Cuba, pese a lo cual los datos son bastante mejores que los que obtuvimos en el presente estudio. Sin embargo, según lo destacan los propios autores, si bien el nivel es bajo para el promedio de los países latinoamericanos, las cifras son elevadas para su contexto nacional, pues en el mismo estudio reportó tan solo un $6,4 \%$ de prevalencia en escolares de la misma edad de escuelas privadas. 


\begin{tabular}{|c|c|c|c|c|c|c|c|c|c|}
\hline \multirow{3}{*}{ Hábitos de higiene bucal } & \multicolumn{4}{|c|}{ FEMENINO } & \multicolumn{4}{|c|}{ MASCULINO } & \multirow{3}{*}{$\begin{array}{l}\text { Sig. } \\
p^{*}\end{array}$} \\
\hline & \multicolumn{2}{|c|}{ No } & \multicolumn{2}{|c|}{$\mathrm{Si}$} & \multicolumn{2}{|c|}{ No } & \multicolumn{2}{|r|}{ Si } & \\
\hline & $\mathbf{n}$ & $\%$ & $n$ & $\%$ & $\mathbf{n}$ & $\%$ & $\mathbf{n}$ & $\%$ & \\
\hline Tiene cepillo dental en casa & 2 & 3,8 & 51 & 96,2 & 0 & 0,0 & 47 & 100,0 & 0,179 \\
\hline Comparte su cepillo dental & 51 & 96,2 & 2 & 3,8 & 46 & 97,9 & 1 & 2,1 & 0,630 \\
\hline Tiene cepillo dental en el colegio & 49 & 92,5 & 4 & 7,5 & 43 & 91,5 & 4 & 8,5 & 0,859 \\
\hline Alguna vez ha asistido al dentista & 10 & 18,9 & 43 & 81,1 & 1 & 2,1 & 46 & 97,9 & 0,008 \\
\hline
\end{tabular}

Tabla 3. HÁBITOS DE HIGIENE BUCAL DE LOS ESCOLARES DE 12 AÑOS DE EDAD INCLUIDOS EN EL ESTUDIO.

Otro estudio, realizado en Chile, mostró que el $92 \%$ de los adolescentes de 12 años en un sector urbano presentaban signos de enfermedad periodontal. ${ }^{19}$ Sin embargo, por tratarse de un estudio efectuado en colegios públicos, podría pensarse que el nivel socioeconómico de estos estudiantes afectaría su estado de salud bucal.

En cuanto a posibles diferencias de prevalencia de enfermedad periodontal en relación con el sexo, los datos obtenidos no permiten concluir que exista una diferencia significativa entre varones y mujeres, coincidiendo con lo reportado en el estudio previo realizado en Ecuador. ${ }^{10}$

La gran mayoría de los escolares estudiados presentó un nivel bajo de severidad de enfermedad periodontal, lo cual quedó reflejado en el bajo promedio del IPR $(0,252)$ correspondiente a tejido de soporte sano. En un estudio realizado en una zona rural de México $^{7}$ se registró un valor superior $(\mathrm{IPR}=0,334)$, que se relaciona con una gingivitis simple, por lo que podríamos inferir que, si bien ambas poblaciones estudiadas presentan prevalencias de enfermedad periodontal elevadas, en promedio el nivel de severidad para ambos países es distinto. Falta aún por dilucidar las causas de esta diferencia.

Llama la atención que, a pesar de que el $86 \%$ de los escolares mencionan cepillarse los dientes de 2 a 3 veces al día (Tabla 2), la prevalencia de enfermedad periodontal es elevada. De tal forma que esta aparente contradicción podría deberse a un cepillado incorrecto por parte de los escolares, o a una limitación inherente al instrumento empleado para obtener la información. En efecto, los datos se calcularon a partir de las respuestas ofrecidas por los escolares, sin ningún tipo de verificación por parte de sus padres o representantes. Por lo tanto, las respuestas podrían no reflejar con exactitud la realidad de cada uno de ellos.

Entre las limitaciones del estudio realizado podemos mencionar el sesgo que significa haber centrado nuestro trabajo exclusivamente en escolares de una zona rural. En efecto, se ha demostrado que pueden existir diferencias significativas al comparar la prevalencia de enfermedad periodontal de escolares de un mismo grupo etario pertenecientes a escuelas públicas y privadas de comunidades rurales y urbanas. ${ }^{16}$ En tal sentido, los resultados obtenidos no pueden ser extrapolados a escolares bajo otras circunstancias. Sin embargo, en vista de la carencia de estudios de este tipo en la región andina, los datos obtenidos se suman a los ya publicados y permitirán tener una visión general de la problemática de salud oral en esta zona.

\section{CONCLUSIONES}

Los resultados del estudio realizado muestran que la prevalencia de enfermedad periodontal en adolescentes de 12 años de una comunidad rural cercana a la ciudad de Cuenca (Azuay, Ecuador) fue elevada (71\%). Estos datos constituyen una referencia epidemiológica importante para futuras investigaciones que aborden problemáticas parecidas. Adicionalmente, considerando la escasez de estudios sobre prevalencia de enfermedades periodontales en el Ecuador, la difusión de estos datos representa un aporte significativo que podría contribuir con el diseño de políticas públicas de intervención en materia de salud oral, que permitan diseñar mejores estrategias de manejo de los recursos disponibles.

CONFLICTO DE INTERESES Las autoras no tienen ningún conflicto de interés.

AGRADECIMIENTOS Las autoras agradecen al Dr. Luis Andrés Yarzábal (Departamento de Investigación, Carrera de odontología. Universidad Católica de Cuenca) por su apoyo en la edición y revisión crítica de este manuscrito y al Dr. Esteban Torracchi por su apoyo en la parte estadística. 


\section{Referencias}

1 Carvajal P. Enfermedades periodontales como un problema de salud pública: el desafío del nivel primario de atención en salud. Rev Clin Periodoncia Implantol Rehabil Oral. 2016; 9(2):177-183.

2 Al-Ghutaimel H, Al-Kahtani S, Al-Duhaimi S. Common Periodontal Diseases of Children and Adolescents. Int $\mathbf{J}$ Dent. 2014.

3 Petersen P, Ogawa H. The global burden of periodontal disease: towards integration with chronic disease prevention and control. Periodontol 2000. 2012; 60: 15-39.

4 Petersen P, Bourgeois D, Ogawa H, Estupinan-Day S, Ndiaye $C$. The global burden of oral diseases and risks to oral health. Bulletin of the World Health Organization. 2005; 83 (9): 661-669.

5 Pareja M, García L, Ysla R, Gutiérrez F, Castañeda C, Cartolín S. Prevalencia y necesidad de tratamiento periodontal en escolares de la institución educativa Andrés Bello. Kiru. 2009; 6(1): 36-38

6 Organización Mundial de la Salud. OMS Web site- Centro de prensa. [Online].; 2012 [cited 2018 Noviembre 15. Available from: https://www.who.int/mediacentre/factsheets/fs318/es/.

7 Hernández J, Tello T, Hernández F, Rosette R. Enfermedad periodontal: prevalencia y algunos factores asociados en escolares de una región mexicana. Rev ADM. 2000; 57(6):222-230.

8 Medina C, Cerrato J, Herrera M. Perfil epidemiológico de la caries dental y enfermedad periodontal, en Nicaragua, año 2005. Revista científica UNAN-León. 2007; 1: 39-46.

9 Cárdenas C, Romero M, Giacaman R. Evolución de la prevalencia de caries y gingivitis en niños de 6 y 12 años de Peralillo, VI Región, entre el año 2000 y el 2010. Rev Clin Periodoncia Implantol Rehabil Oral. 2011;4(3):102-105.

10 Jimeno M, Vásquez A. Prevalencia de enfermedad periodontal en escolares de 12 años en la parroquia Machángara, Cuenca 2016. Oactiva. 2017; 2(3): 11-16.

11 Vandenbroucke J, Von Elm E, Altman D, Gøtzsche P, Mulrow $\mathrm{C}$, Pocock $\mathrm{S}$ et al. Mejorar la comunicación de estudios obseervacionales en epidemiología (STROBE): explicación y elaboración. Gac Sanit. 2009; 23 (2): 158.e1-158.e28.

12 Russell A. A system of classification and scoring for prevalence surveys of periodontal disease. J. Dent. Res. 1956; 35 (3): 350-359.

13 Sanz M. 1er Workshop Ibérico Control de placa e higiene bucodental. Madrid: Ergon-Sociedad Española de Periodoncia y Osteointegración; 2003.

14 Deepa D, Jain G. Assessment of periodontal health status in postmenopausal women visiting dental hospital from in and around Meerut city: Cross-sectional observational Study. J Mid-life Health 2016; 7:175-179.

15 Agresti A. An Introduction to Categorical Data Analysis. 2da ed, New York. 2007:38.
16 Freire M, Reis S, Gonçalves M, Balbo P, Leles C. Condição de saúde bucal em escolares de 12 anos de escolas públicas e privadas de Goiânia, Brasil. Rev Panam Salud Pública. 2010; 28(2): 86-91.

17 Perdomo C, Mediaceja N, Junco R, Ramírez G. Estado de salud bucal en pobladores del municipio de Palma Soriano. Medisan. 2016; 20(5):690

8 Sosa M. Programa nacional de atención estomatológica integral. Habana; 2009

19 Becker J, Millatureo D, Juárez-Membreño I, Lagos A. Necesidad de tratamiento periodontal en adolescentes de 12 años de colegios municipalizados en Valdivia-Chile 2014: estudio transversal. Rev Clin Periodoncia Implantol Rehabil Oral. 2016;9(3):259-263.

Recibido: 12 de octubre de 2018. Aceptado: 31 de noviembre de 2018. 
